\title{
Overview of SND hadronic cross section measurements
}

\author{
T.V.Dimova ${ }^{1,2, \star}$, M.N.Achasov ${ }^{1,2}$, V.M.Aulchenko ${ }^{1,2}$, A.Yu.Barnyakov ${ }^{1,2}$, K.I.Beloborodov ${ }^{1,2}$, \\ A.V.Berdyugin ${ }^{1,2}$, D.E.Berkaev ${ }^{1,2}$, A.G.Bogdanchikov ${ }^{1}$, A.A.Botov ${ }^{1}$, V.P.Druzhinin ${ }^{1,2}$, \\ E.B.Ganeev ${ }^{1}$, V.B.Golubev ${ }^{1,2}$, L.V.Kardapoltsev ${ }^{1,2}$, A.S.Kasaev ${ }^{1}$, A.G.Kharlamov ${ }^{1,2}$, \\ A.N.Kirpotin 1, I.A.Koop ${ }^{1,2,3}$, L.A.Korneev ${ }^{1,2}$, A.A.Korol ${ }^{1,2}$, S.V.Koshuba1, D.P.Kovrizhin ${ }^{1,2}$, \\ A.S.Kupich ${ }^{1,2}$, R.A.Litvinov ${ }^{1,2}$, K.A.Martin ${ }^{1}$, N.A.Melnikova ${ }^{1,2}$, N.Yu.Muchnoi ${ }^{1,2}$, A.E.Obrazovsky $^{1}$, \\ E.V.Pakhtusova1, K.V.Pugachev ${ }^{1,2}$, Yu.A.Rogovsky ${ }^{1,2}$, E.V.Rogozina1, S.I.Serednyakov ${ }^{1,2}$, \\ A.I.Senchenko ${ }^{1}$, D.A.Shtol ${ }^{1,2}$, D.B.Shwartz ${ }^{1,2}, \quad$ Z.K.Silagadze ${ }^{1,2}$, I.K.Surin ${ }^{1}$, Yu.V.Usov ${ }^{1}$, \\ A.V.Vasiljev ${ }^{1,2}$, and V.N.Zhabin ${ }^{1,2}$ \\ ${ }^{1}$ Budker Institute of Nuclear Physics, Novosibirsk, 630090, Russia \\ ${ }^{2}$ Novosibirsk State University, 630090 Novosibirsk, Russia \\ ${ }^{3}$ Novosibirsk State Technical University, Novosibirsk, 630092, Russia
}

\begin{abstract}
At the end of 2016 the SND detector resumed data taking at the upgraded VEPP-2000 $e^{+} e^{-}$collider. The analysis of data accumulated in 2010-2013 with an integrated lumonosity of $70 \mathrm{pb}^{-1}$ is continuing. Recent results on measurements of various hadronic cross sections are presented.
\end{abstract}

\section{Introduction}

The SND [1-4] is the general purpose nonmagnetic detector. Its main part is a spherical three-layer electromagnetic calorimeter containing $1640 \mathrm{NaI}(\mathrm{Tl})$ crystals. Directions of charged particles are measured by a tracking system based on a nine-layer drift chamber. The particle identification is provided by $d E / d x$ measurements in the tracking system and a system of aerogel Cherenkov counters. Outside the calorimeter a muon detector is located.

SND collected data at the VEPP-2M [5] and VEPP2000 [6] $e^{+} e^{-}$colliders. At VEPP-2M, data with an integrated luminosity of about $30 \mathrm{pb}^{-1}$ were recorded in 1996-2000 in the energy range 0.4$1.4 \mathrm{GeV}$. At VEPP2000, a wider energy interval, $0.3-2.0 \mathrm{GeV}$, is studied. A $69 \mathrm{pb}^{-1}$ data sample was collected during 2010-2013. At the end of 2016 year after finishing of upgrade VEPP2000 resumed operation. About $50 \mathrm{pb}^{-1}$ of integrated luminosity has been accumulated during first data taking run.

The main goal of the SND experiments is careful measurement of exclusive hadronic cross sections below $2 \mathrm{GeV}$, which are, in particular, needed for the Standard Model calculation of the muon $(g-2)$ and running $\alpha_{Q E D}$. Here we present new results on measurement of four exclusive cross sections and three rare processes.

\footnotetext{
^e-mail: baiert@inp.nsk.su
} 


\section{Precise cross-section measurements}

\section{$2.1 e^{+} e^{-} \rightarrow \pi^{0} \gamma$ cross section}

The $e^{+} e^{-} \rightarrow \pi^{0} \gamma$ cross section is the third largest cross section (after $e^{+} e^{-} \rightarrow \pi^{+} \pi^{-}$and $\pi^{+} \pi^{-} \pi^{0}$ ) below $1 \mathrm{GeV}$. From analysis of the $e^{+} e^{-} \rightarrow \pi^{0} \gamma$ data in the vector meson dominance (VMD) model, the widths of vector-meson radiative decays are extracted, which are widely used in phenomenological models. The most accurate data on this process were obtained in experiments at the VEPP-2M $e^{+} e^{-}$ collider with the SND [7, 8] and CMD-2 [9] detectors. The SND results [7, 8] are based on about 25\% of data collected at VEPP-2M. Here we present a new analysis [10] using the full SND@ VEPP-2M data sample. Also preliminary results based on data accumulated by SND detector at VEPP-2000 collider in the energy range above $\phi$ meson mass are shown.

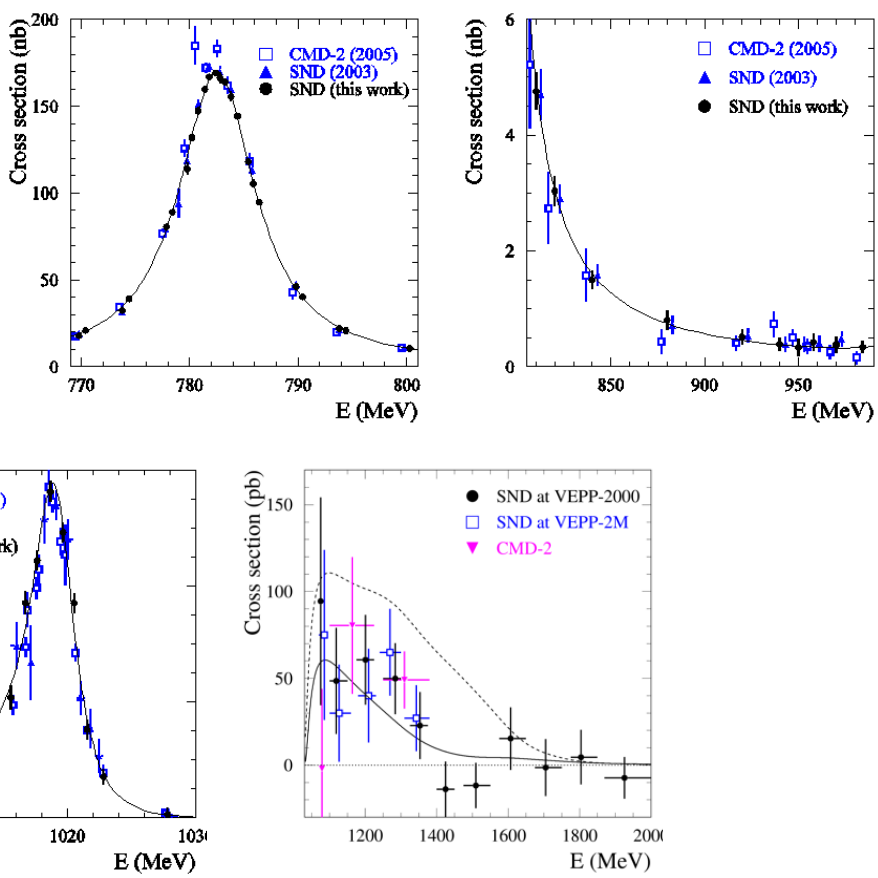

Figure 1. The $e^{+} e^{-} \rightarrow \pi^{0} \gamma$ cross section measured by SND using the full VEPP-2M data sample in comparison with the previous most accurate measurements. The curve is the result of the VMD fit. Only statistical errors are shown. The systematic errors are 3.2\%, 3\%, and 6\% for SND (2000), SND (2003), and CMD-2 (2005) data, respectively. Our systematic uncertainty at the $\omega$ and $\phi$ peaks is $1.4 \%$. In the energy range above $\phi$ meson mass the preliminary results based on data accumulated at VEPP-2000 are shown.

The measured $e^{+} e^{-} \rightarrow \pi^{0} \gamma$ cross section shown in Fig. 1 agrees with previous SND [7, 8] and CMD-2 [9] measurements within the systematic uncertainties, but is significantly more precise.

\section{$2.2 e^{+} e^{-} \rightarrow K^{+} K^{-}$cross section}

In this measurement charged kaon identification is based on information from the aerogel threshold Cherenkov counters [11]. The measured cross section of the $e^{+} e^{-} \rightarrow K^{+} K^{-}$process in comparison 
with BABAR data [12] is shown in Fig.2 (left). The complex energy dependence of the cross section is explained by interference of the amplitudes of all isoscalar and isovector resonances located in the energy region under study. Our measurement is in good agreement with BABAR data and has comparable or better accuracy (Fig.2 (right)).
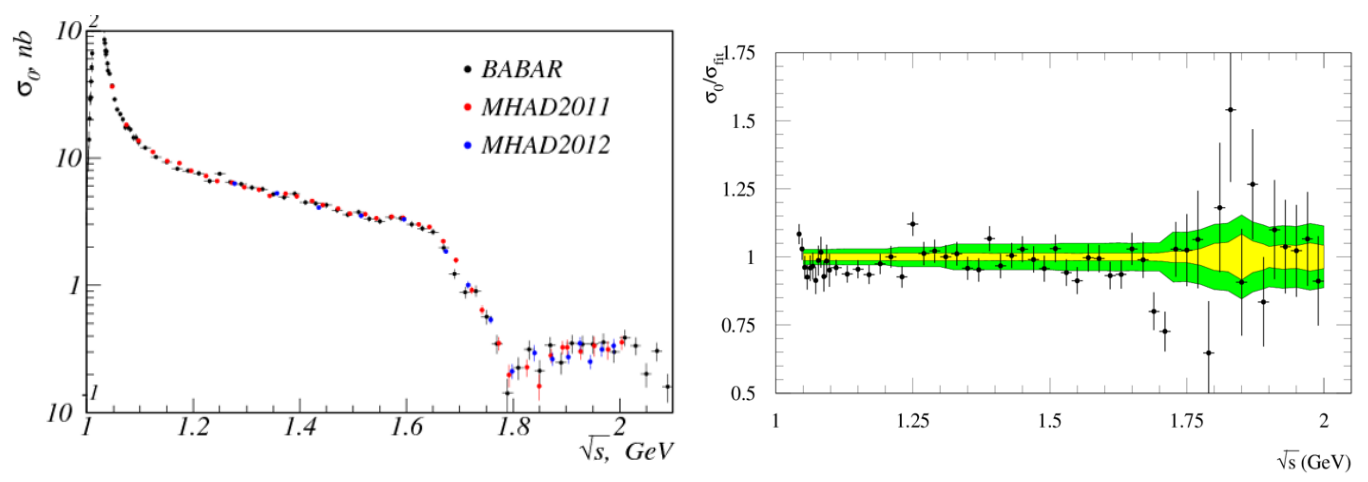

Figure 2. Left panel: The $e^{+} e^{-} \rightarrow K^{+} K^{-}$cross section measured by SND at VEPP2000 and in the BABAR experiment [12]. Right panel: The relative difference between the $e^{+} e^{-} \rightarrow K^{+} K^{-}$cross sections measured by BABAR and the fit to the SND data. The SND and BABAR systematic uncertainties are shown by the yellow and green bands, respectively.

\section{$2.3 e^{+} e^{-} \rightarrow \omega \pi^{0} \rightarrow \pi^{0} \pi^{0} \gamma$ cross section}

The update our previous measurement of the $e^{+} e^{-} \rightarrow \omega \pi^{0}$ cross section [13] based on the full SND data set collected at VEPP-2000 is presented in Fig. 3 (left)in comparison with the SND at VEPP$2 \mathrm{M}$ result [14, 15], CMD-2 [16] and DM2 [17] data. The cross-section energy dependence is well described by contributions of the $\rho, \rho(1450)$ and $\rho(1700)$ resonances. The transition form factor $F_{\omega \pi \gamma}$ for $\gamma^{*} \rightarrow \omega \pi^{0}$ vertex $F_{\omega \pi \gamma}$ obtained from the measured cross section is shown in Fig.3(right). Below $0.7 \mathrm{GeV}$ the same form factor measured in the $\omega \rightarrow \pi^{0} \mu^{+} \mu^{-}$decay [18] is shown. The solid curve represents the results of the VMD prediction with the parameters obtained from our cross section fit. The dashed curve shows the $\rho$ (770) contribution only. One can see that the data from $e^{+} e^{-}$annihilation and $\omega \rightarrow \pi^{0} \mu^{+} \mu^{-}$decay cannot be described within the VMD model simultaneously.

\section{$2.4 e^{+} e^{-} \rightarrow \omega \pi^{0} \rightarrow \pi^{+} \pi^{-} \pi^{0} \pi^{0}$ cross section}

Preliminary results on cross section of $e^{+} e^{-} \rightarrow \omega \pi^{0} \rightarrow \pi^{+} \pi^{-} \pi^{0} \pi^{0}$ process in the energy range 1-2 $\mathrm{GeV}$ in comparison with previous SND measurements $[13,19,20]$ are presented (Fig.4 (left)). The statistical error varies between 2 and 16\%, while systemaic error between 1 and $9 \%$ depending on the energy. The cross section is fitted using contributions of the $\rho, \rho(1450)$ and $\rho(1700)$ resonances.

\section{Rare processes}

\section{$3.1 e^{+} e^{-} \rightarrow K^{+} K^{-} \eta$ cross section}

This process is studied in the $\eta \rightarrow \gamma \gamma$ decay mode. The measured cross section in comparison with BABAR data is shown in Fig.4 (right). The fit to the cross section data is performed in hypothesis that 

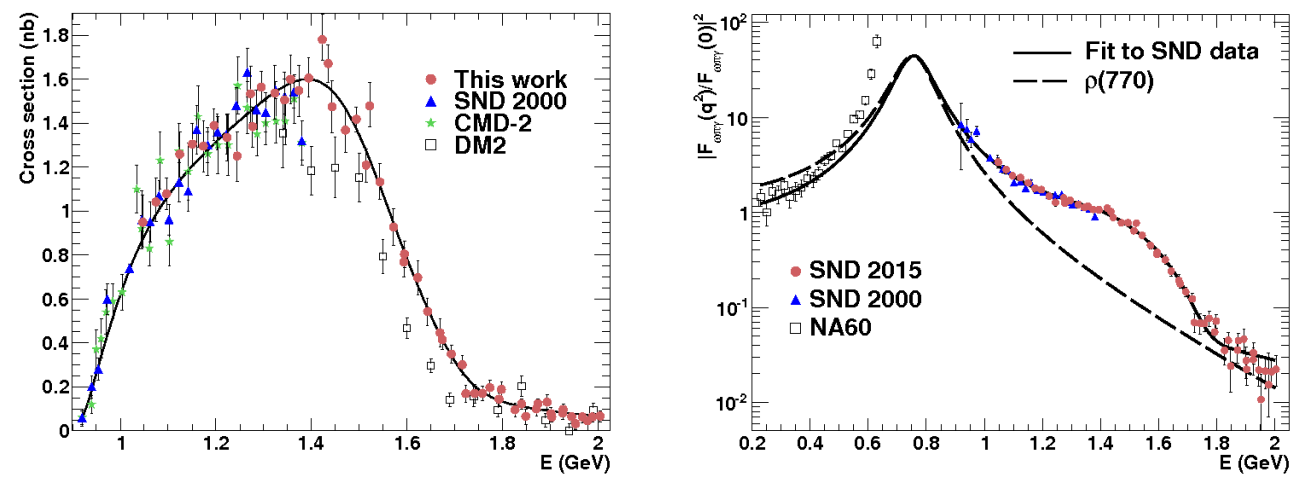

Figure 3. Left panel: The $e^{+} e^{-} \rightarrow \omega \pi^{0} \rightarrow \pi^{0} \pi^{0} \gamma$ cross section measured by SND at VEPP2000 and VEPP$2 \mathrm{M}[14,15]$, and by CMD-2[16] and DM2[17] experiments. Only statistical errors are shown. The curve is the result of the fit to SND 2000 and SND 2013. Right panel: The $\gamma^{*} \rightarrow \omega \pi^{0}$ transition form factor. The solid curve is the result of the fit to the SND data with the VMD model. The $\rho(770)$ contribution is shown by the dashed curve.

main mechanism of this reaction is $e^{+} e^{-} \rightarrow \phi(1680) \rightarrow \phi(1020) \eta$. The result of the fit is in agreement with the data.
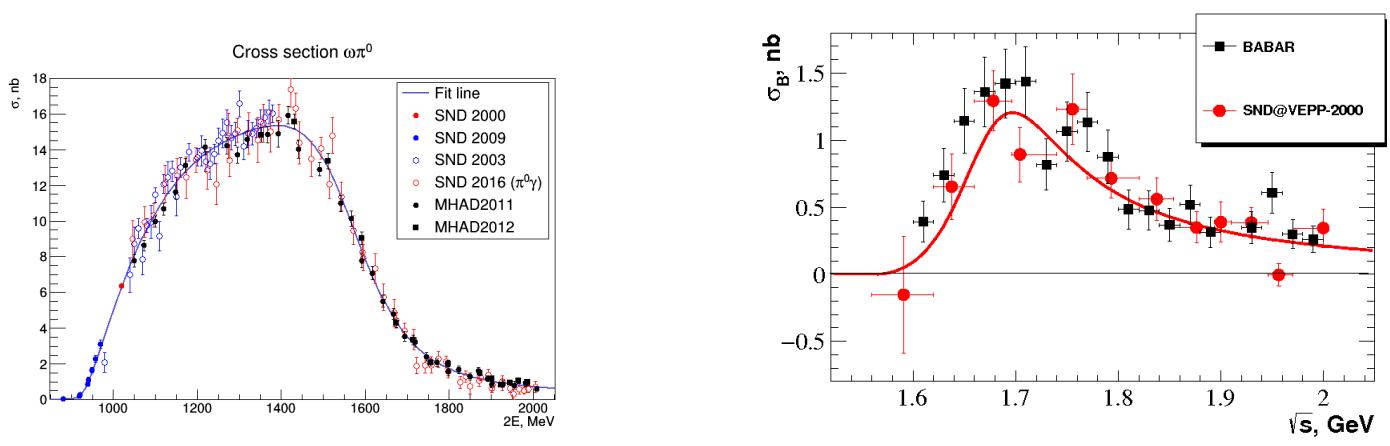

Figure 4. Left panel: The $e^{+} e^{-} \rightarrow \omega \pi^{0} \rightarrow \pi^{+} \pi^{-} \pi^{0} \pi^{0}$ cross section measured by SND at VEPP2000 and VEPP$2 \mathrm{M}([19,20])$ in comparison with $e^{+} e^{-} \rightarrow \omega \pi^{0} \rightarrow \pi^{0} \pi^{0} \gamma$ [13] cross section. Right panel: The $e^{+} e^{-} \rightarrow K^{+} K^{-} \eta$ cross section measured by SND at VEPP-2000 and in the BABAR experiment [24]. The solid curve is the result of the fit described in the text.

\section{$3.2 e^{+} e^{-} \rightarrow \pi^{+} \pi^{-} \pi^{0} \eta$ cross section}

The process $e^{+} e^{-} \rightarrow \pi^{+} \pi^{-} \pi^{0} \eta$ has complex internal structure. Our preliminary study show that there are at least four mechanisms for this reaction: $\omega \eta, \phi \eta, a_{0}(980) \rho$, and structureless $\pi^{+} \pi^{-} \pi^{0} \eta$. The known $\omega \eta$ and $\phi \eta$ contributions explain about $50-60 \%$ of the cross section below $1.8 \mathrm{GeV}$. Above 1.8 $\mathrm{GeV}$ the dominant mechanism is $a_{0} \rho$. The preliminary result on the $e^{+} e^{-} \rightarrow \pi^{+} \pi^{-} \pi^{0} \eta$ cross section is shown in Fig. 5 (left). The cross section for the subprocess $e^{+} e^{-} \rightarrow \omega \eta$ is measured separately [21] 
and shown in Fig. 5 (right) in comparison with the BABAR measurement [22]. Our results have better accuracy and disagree with the BABAR data at $E>1.6 \mathrm{GeV}$.
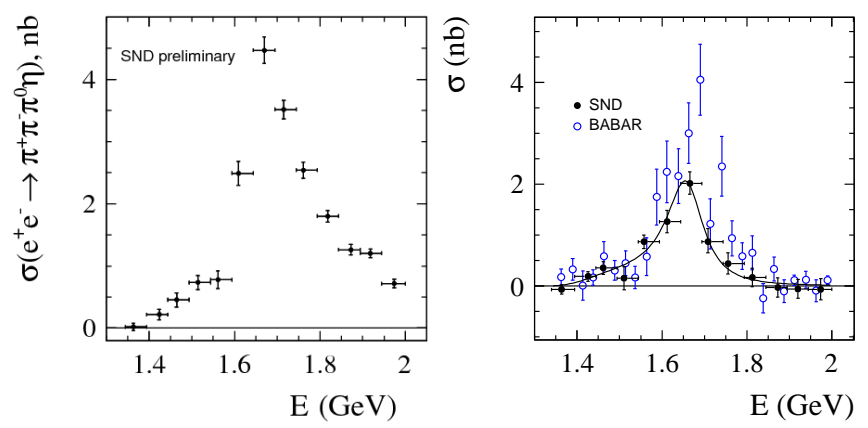

Figure 5. Left panel: The $e^{+} e^{-} \rightarrow \pi^{+} \pi^{-} \pi^{0} \eta$ cross section measured by SND. Right panel: The $e^{+} e^{-} \rightarrow \omega \eta$ cross section measured by SND in comparison with BABAR data [22]. The curve is the result of the VMD fit.

\section{$3.3 e^{+} e^{-} \rightarrow \omega \pi^{0} \eta$ cross section}

The process $e^{+} e^{-} \rightarrow \omega \pi^{0} \eta$ is studied in the seven-photon final state [23]. Events of the $e^{+} e^{-} \rightarrow \pi^{0} \pi^{0} \eta \gamma$ process are selected. The analysis of their $\pi^{0} \gamma$ invariant-mass distribution (Fig.6 (left) shows the dominance of the $\omega \pi^{0} \eta$ intermediate state. Figure 6 (middle) shows the $\pi^{0} \eta$ mass distribution for selected $\omega \pi^{0} \eta$ events, which is well described by the model of the $\omega a_{0}(980)$ intermediate state. The measured $e^{+} e^{-} \rightarrow \omega \pi^{0} \eta$ cross section is shown in Fig. 6 (right).
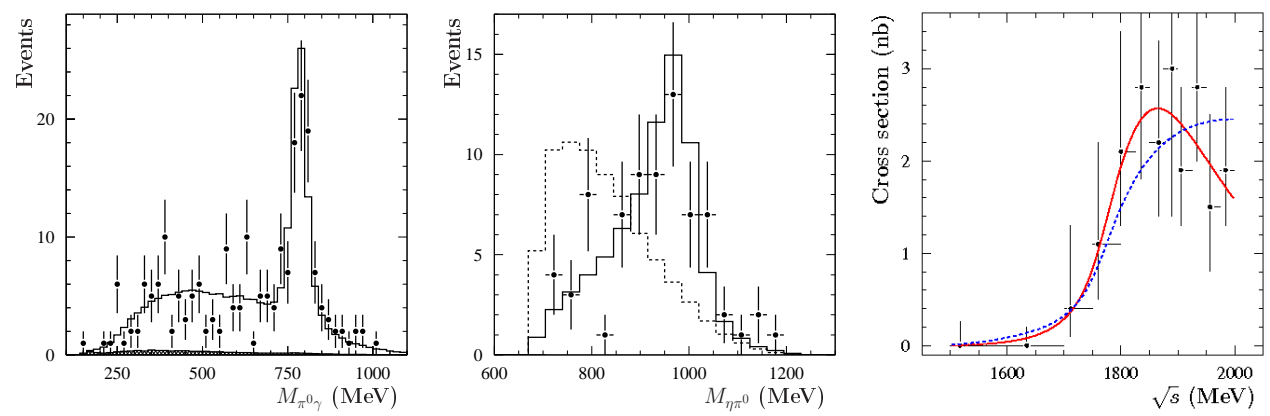

Figure 6. Left panel: The $\pi^{0} \gamma$ invariant mass spectrum which shows the dominance of the $\omega \pi^{0} \eta$ intermediate state. Middle panel: The $\eta \pi^{0}$ invariant mass spectrum for selected $e^{+} e^{-} \rightarrow \omega \eta \pi^{0}$ events. The solid histogram represents $e^{+} e^{-} \rightarrow \omega a_{0}(980)$ simulation, while the dashed histogram represents the $\omega \eta \pi^{0}$ phase-space simulation. Right panel: The $e^{+} e^{-} \rightarrow \omega \eta \pi^{0}$ cross section measured by SND. The solid (dashed) curve shows the result of the fit with (without) a resonance contribution.

\section{Conclusions}

During 2010-2013 the SND detector accumulated about $70 \mathrm{pb}^{-1}$ of integrated luminosity at the VEPP$2000 e^{+} e^{-}$collider in the c.m. energy range $0.3-2 \mathrm{GeV}$. Data analysis on hadron production is in 
progress. The results of analysis of processes $e^{+} e^{-} \rightarrow \pi^{0} \gamma, \omega \pi^{0}, K^{+} K^{-}, \pi^{+} \pi^{-} \pi^{0} \eta, \omega \pi^{0} \eta, K^{+} K^{-} \eta$ are presented. After VEPP-2000 upgraded the data taking runs are resumed with a goal of $\sim 1 \mathrm{fb}^{-1}$ of integrated luminosity.

Acknowledgments: This work is supported in part by the RFBR grants 16-02-00327, 16-02-00014, 15-0201037, and 15-02-03391. Part of this work related to the photon reconstruction algorithm in the electromagnetic calorimeter is supported by the Russian Science Foundation (project No. 14-50-00080).

\section{References}

[1] M. N. Achasov et al., Nucl. Instrum. Methods Phys. Res., Sect. A 598, 31 (2009)

[2] V. M. Aulchenko et al., Nucl. Instrum. Methods Phys. Res., Sect. A 598, 102 (2009)

[3] A. Y. Barnyakov et al., JINST 9, C09023 (2014)

[4] V. M. Aulchenko et al., Nucl. Instrum. Methods Phys. Res., Sect. A 598, 340 (2009)

[5] I. A. Koop et al., in Proceedings of the Workshop on Physics and Detectors for DAPHNE, Frascati, Italy, 1999 (Frascati, 1999), p. 393

[6] A. Romanov et al., in Proceedings of Particle Accelerator Conference PAC 2013, Pasadena, CA USA, 2013, p. 14

[7] M. N. Achasov et al. (SND Collaboration), Eur. Phys. J. C 12, 25 (2000)

[8] M. N. Achasov et al. (SND Collaboration), Phys. Lett. B 559, 171 (2003)

[9] R. R. Akhmetshin et al. (CMD-2 Collaboration), Phys. Lett. B 605, 26 (2005)

[10] M. N. Achasov et al. (SND Collaboration), Phys. Rev. D 93, 092001 (2016)

[11] A.Yu.Barnyakov, M.Yu.Barnyakov, K.I.Beloborodov et al., JINST 9 C09023 (2014)

[12] J. P. Lees et al. (BaBar Collaboration), Phys. Rev. D 88, 032013 (2013)

[13] M.N.Achasov et al.. (SND Collaboration), Phys. Rev. D 94, 112001 (2016).

[14] M. N. Achasov et al. (SND Collaboration), Phys. Lett. B 486, 29 (2000)

[15] V. M. Aulchenko et al.(SND Collaboration), Zh. Eksp. Teor. Fiz. 117, 1067 (2000) [J. Exp.

Theor. Phys.90, 927(2000)].

[16] R. R. Akhmetshin et al. (CMD-2 Collaboration), Phys. Lett.B 562, 173 (2003).

[17] D. Bisello et al. (DM2 Collaboration), Nucl. Phys. Proc. Suppl.21, 111 (1991).

[18] R.Arnaldi, K. Banicz, J. Castor et al., Phys. Lett. B 677, 260 (2009).

[19] M. N. Achasov et al.(SND Collaboration), J. Exp. Theor. Phys.96, 789(2003).

[20] M. N. Achasov et al.(SND Collaboration), J. Exp. Theor. Phys.109, 379(2009).

[21] M. N. Achasov et al. (SND Collaboration), Phys. Rev. D 94, 092002 (2016)

[22] B. Aubert et al. (BABAR Collaboration), Phys. Rev. D 73, 052003 (2006)

[23] M. N. Achasov et al. (SND Collaboration), Phys. Rev. D 94, 032010 (2016)

[24] B. Aubert et al. Phys. Rev. D 77, 092002 (2008) 